\title{
Cyclin D1 immunohistochemical staining to separate benign from malignant mesothelial proliferations
}

\author{
Jennifer Pors ${ }^{1} \cdot$ Julia Naso $^{1} \cdot \mathrm{Kyra}_{\mathrm{Berg}}{ }^{1} \cdot$ Andrew Churg $^{1}$
}

Received: 24 August 2019 / Revised: 26 October 2019 / Accepted: 26 October 2019 / Published online: 4 November 2019

(c) The Author(s), under exclusive licence to United States \& Canadian Academy of Pathology 2019

\begin{abstract}
The separation of benign from malignant mesothelial proliferations is a morphologically difficult problem. Mutations/ deletions of components of the Hippo pathway are frequent in malignant mesotheliomas, and one downstream effect of aberrant Hippo signaling is increased production of cyclin D1. We examined expression of cyclin D1 nuclear staining in two tissue microarrays containing 52 reactive epithelial mesothelial proliferations, 51 reactive spindle cell mesothelial proliferations, 54 epithelial mesotheliomas, and 22 sarcomatous/desmoplastic mesotheliomas. When present, cyclin D1 staining was always strong, hence the arrays were scored as $0,1-25 \%, 26-50 \%, 51-75 \%$, and $76-100 \%$ staining. Both arrays showed a similar pattern. Reactive epithelial proliferations generally showed no staining (42/52 cases) or $1-25 \%$ staining (10/52 cases) with no cases showing $>25 \%$ staining. Overall for reactive epithelial proliferations the maximum staining was $14.8 \%$ and mean $1.1 \pm 2.9 \%$. For epithelial mesotheliomas $39 / 54(72 \%)$ cases demonstrated $>25 \%$ staining, with $8 / 54$ in the $26-50 \%$ staining range, $9 / 54$ in the $51-75 \%$ range, and $22 / 54$ in the $>75 \%$ range. Combinations of staining using cyclin D1 $>50 \%$ plus BAP1 or MTAP loss in epithelial mesotheliomas produced about a $10 \%$ increase in sensitivity. Reactive spindle cell proliferations showed a broader range of staining with $27 / 51$ in the 1-25\% range, 5/51 in the 26-50\% range, and $1 / 51>50 \%$. Eleven of 22 sarcomatous/desmoplastic mesotheliomas scored $50 \%$ or greater. We conclude that for epithelial mesothelial proliferations, the finding of $>50 \%$ of tumor cells staining supports a diagnosis of epithelial mesothelioma with $100 \%$ specificity but only modest (57\%) sensitivity.
\end{abstract}

\section{Introduction}

The separation of benign from malignant mesothelial proliferations is crucial to patient care, but is, morphologically, often a difficult problem. In the last 10 years a variety of new ancillary techniques have been developed that aid in this separation, including BAP1, MTAP, and 5-hydroxymethyl cytosine immunohistochemical staining; and $C D K N 2 A$ and $N F 2$ fluorescence in situ hybridization (FISH), but each has drawbacks in terms of histologic subtype of mesothelioma (epithelial vs. sarcomatous), location (thorax vs. abdomen)

Supplementary information The online version of this article (https:// doi.org/10.1038/s41379-019-0411-9) contains supplementary material, which is available to authorized users.

Andrew Churg achurg@mail.ubc.ca

1 Department of Pathology, Vancouver General Hospital and University of British Columbia, Vancouver, BC, Canada of the mesothelial process, or technical skills required and ease of interpretation of the results [1-7].

One of the recurring genetic abnormalities in malignant mesothelioma is mutation or deletion of genes in the Hippo signaling pathway [7-10]. Attempts to exploit this pathway for diagnostic purposes have, thus far, had limited success (see Discussion). Aberrant functioning of the Hippo pathway leads, among other things, to overproduction of cyclin D1 (see Discussion) $[8,9]$. Here we show that immunostaining for cyclin D1 can be used in the separation of benign from malignant mesothelial processes.

\section{Materials and methods}

\section{Case selection and tissue microarray preparation}

This study was approved by the Clinical Research Ethics Board of the University of British Columbia and Vancouver Coastal Health. Cases of malignant mesothelioma and reactive mesothelial proliferations were acquired retrospectively 
from the anatomical pathology archives at Vancouver General Hospital. All mesotheliomas were confirmed by appropriate immunohistochemical staining. Reactive mesothelial proliferations were confirmed by clinical follow-up. Two tissue microarrays were used. The first contained 16 reactive epithelial mesothelial proliferations, 15 reactive spindle cell mesothelial proliferations, 27 epithelial malignant mesotheliomas, and 8 sarcomatous ldesmoplastic mesotheliomas. The second tissue microarray contained 36 reactive epithelial mesothelial proliferations, 36 reactive spindle cell mesothelial proliferations, 27 epithelial malignant mesotheliomas, and 14 sarcomatous desmoplastic mesotheliomas.

\section{Immunohistochemistry}

Immunohistochemical staining for cyclin D1 was performed on a Dako Omnis automated IHC instrument using Dako anti-cyclin D1 rabbit monoclonal clone EP12 (as supplied by the manufacturer) with the Dako EnVision $^{\mathrm{TM}}$ FLEX + detection system. BAP1 and MTAP antibody staining was performed as previously described [3].

For cyclin D1 only nuclear staining was assessed. Because staining, when present, was always strong, staining scores were based on proportion of cells staining (0, 1-25\%; 26-50\%; 51-75\%; 76-100\%). For BAP1 staining was assessed as nuclear loss or retention; for MTAP staining was assessed as cytoplasmic loss or retention only and nuclear staining was ignored [3].

Statistical analysis differences in the distribution of staining were calculated by Chi-square analysis with Yates correction or Fisher's exact test. The sensitivity, specificity, positive predictive value, and negative predictive value for cyclin D1 in distinguishing benign mesothelial proliferations from epithelial malignant mesotheliomas and sarcomatous Idesmoplastic malignant mesotheliomas was determined using an online statistical calculator (https://www.medcalc. org/calc/diagnostic_test.php) with $>50 \%$ cyclin D1 staining set as the cut-off for epithelial malignant mesotheliomas and $>50 \%$ or $75 \%$ staining set as the cut-off for sarcomatous Idesmoplastic malignant mesotheliomas.

\section{Results}

\section{Cyclin D1 staining in normal tissue}

Normal lung and pleural tissue as well as reactive pleural tissue usually, but not invariably, showed staining in vascular endothelial cells, which can serve as a useful positive internal control; however, vascular staining was often patchy (Fig. 1). In some cases, normal lung tissue also showed staining of alveolar lining cells, but this was inconsistent.

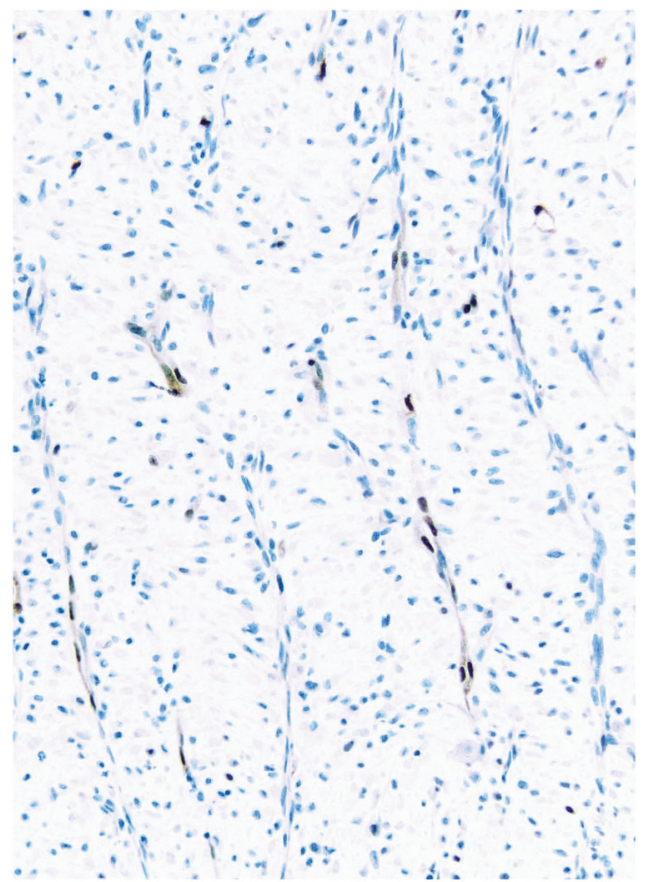

Fig. 1 Prominent cyclin D1 staining in vascular endothelium in a case of organizing pleuritis. Although staining is strong when present (and serves as a good internal control when positive), it is very patchy. In this case the spindled mesothelial cells are negative

Fat cells in the pleura sometimes stained but again this staining was inconsistent.

\section{Cyclin D1 staining in TMAs}

Table 1 shows the staining results in the two individual microarrays and for both combined, which together totaled 52 reactive epithelial mesothelial proliferations, 51 reactive spindle cell mesothelial proliferations, 54 epithelial malignant mesotheliomas, and 22 sarcomatous/desmoplastic malignant mesotheliomas. The combined data are shown graphically in Fig. 2. The distribution of staining was similar in both tissue microarrays. Forty two of 52 reactive epithelial mesothelial proliferations showed no staining at all (Fig. 3) and 10/52 showed staining in the 1-25\% range. No case of reactive epithelial mesothelial proliferations showed staining in over $25 \%$ of cells, and in most of the positive cases in the $1-25 \%$ group, staining was only a few percent of cells; overall the mean staining percent for reactive epithelial mesothelial proliferations was $1.1 \pm 2.8$ (mean $\pm \mathrm{SD}$ ), median 0 , and range 0-14.8\% . Reactive spindle cell mesothelial proliferations tended to show more staining, with $27 / 51(53 \%)$ in the $1-25 \%$ range, $5 / 51(10 \%)$ in the $26-50 \%$ range, and $1 / 51$ cases in the $51-75 \%$ range (Fig. 3).

Overall $39 / 54$ (72\% of total cases) of epithelial malignant mesotheliomas showed $>25 \%$ staining, with $8 / 54$ 
Table 1 Cyclin D1 scores (as \% staining)

\begin{tabular}{lrrrrrr}
\hline Score & 0 & $1-25 \%$ & $26-50 \%$ & $51-75 \%$ & $76-100 \%$ & $N$ \\
\hline Tissue microarray 1 & & & & & & \\
$\quad$ Reactive epithelial proliferations & 10 & 6 & 0 & 0 & 0 & 16 \\
Reactive spindle cell proliferations & 4 & 8 & 3 & 0 & 0 & 15 \\
$\quad$ Epithelial malignant mesotheliomas & 5 & 5 & 2 & 3 & 12 & 27 \\
$\quad$ Sarcomatous/desmoplastic malignant mesotheliomas & 1 & 1 & 1 & 1 & 4 & 8 \\
Tissue microarray 2 & & & & & & \\
Reactive epithelial proliferations & 32 & 4 & 0 & 0 & 0 & 36 \\
Reactive spindle cell proliferations & 14 & 19 & 2 & 1 & 0 & 36 \\
Epithelial malignant mesotheliomas & 1 & 4 & 6 & 6 & 10 & 27 \\
$\quad$ Sarcomatous/desmoplastic malignant mesotheliomas & 4 & 4 & 0 & 0 & 6 & 14 \\
Combined tissue microarrays & & & & & & \\
Reactive epithelial proliferations & 42 & 10 & 0 & 0 & 0 & 52 \\
Reactive spindle cell proliferations & 18 & 27 & 5 & 1 & 0 & 51 \\
Epithelial malignant mesotheliomas & 6 & 9 & 8 & 9 & 22 & 54 \\
Sarcomatous/desmoplastic malignant mesotheliomas & 5 & 5 & 1 & 1 & 10 & 22 \\
\hline
\end{tabular}

Chi-square with Yates correction for combined microarrays

Reactive epithelial proliferations vs. epithelial malignant mesotheliomas Chi-square $=63.1, \mathrm{df}=4, p<1 \times$ $10^{-7}$

Reactive spindle cell proliferations vs. sarcomatous/desmoplastic malignant mesotheliomas Chi-square $=$ $23.1 \mathrm{df}=4, p=0.0001$

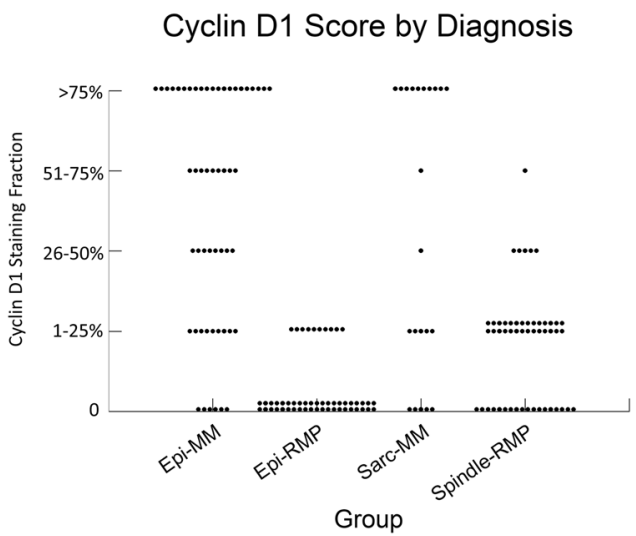

Fig. 2 Cyclin D1 scores from both TMAs combined, by diagnosis. Epi-MM epithelial malignant mesotheliomas. Epi-RMP reactive epithelial mesothelial proliferations. Sarc-MM sarcomatous/desmoplastic mesotheliomas. Spindle-RMP reactive spindle cell mesothelial proliferations

(15\% of total cases) in the $26-50 \%$ staining range, $9 / 54$ (17\% of total cases) in the $51-75 \%$ staining range, and $22 /$ 54 ( $41 \%$ of total cases) in the $>75 \%$ staining range (Fig. 3 ). The pattern of staining for sarcomatous/desmoplastic malignant mesotheliomas was also similar in both TMAs with $1 / 22(5 \%)$ demonstrating $26-50 \%$ staining, $1 / 22$ showing $51-75 \%$ staining, and $10 / 22(45 \%)>75 \%$ staining for the combined microarrays (Fig. 3). Seven of the 22 sarcomatous/desmoplastic malignant mesotheliomas were desmoplastic and these showed a range of staining with one case completely negative, three in the
$1-25 \%$ range, two in the $50-75 \%$ range, and one in the greater than $75 \%$.

For statistical purposes the two microarrays were combined. The chi-square value for epithelial malignant mesotheliomas compared with reactive epithelial mesothelial proliferations was 63.1 with $\mathrm{df}=4$, and $p<1 \times 10^{-7}$. For sarcomatous/desmoplastic malignant mesotheliomas versus reactive spindle cell mesothelial proliferations Chi-square $=23.1, \mathrm{df}=4, p=0.0001$.

At a 50\% cutoff, Cyclin D1 staining had a sensitivity of $57 \%$, a specificity of $100 \%$, a positive predictive value of $100 \%$, and a negative predictive value of $69 \%$ in distinguishing epithelial malignant mesotheliomas from reactive epithelial mesothelial proliferations. At a 50\% cutoff, Cyclin D1 staining had a sensitivity of $50 \%$, a specificity of $98 \%$, a positive predictive value of $92 \%$, and a negative predictive value of $82 \%$ in distinguishing sarcomatous/ desmoplastic malignant mesotheliomas from reactive spindle cell mesothelial proliferations. At a $75 \%$ cutoff, cyclin D1 staining had a sensitivity of $45 \%$, a specificity of $100 \%$, a positive predictive value of $100 \%$, and an negative predictive value of $81 \%$ for distinguishing sarcomatous/desmoplastic malignant mesotheliomas from reactive spindle cell mesothelial proliferations.

\section{BAP1 and MTAP staining}

BAP1 staining was evaluable in 37 epithelial malignant mesotheliomas cases from the combined microarrays 
Fig. 3 a Cyclin D1 stain showing no staining in the reactive surface epithelial mesothelial cells and very rare staining of a spindle cell nucleus in the underlying reactive spindle mesothelial cells. Arrows points out vessels with endothelial staining. b Cyclin D1 stain of a reactive spindle cell mesothelial proliferation showing distinct nuclear staining in some of the spindle cells. c Cyclin D1 stain of an epithelial malignant mesothelioma showing nuclear staining in $>75 \%$ of tumor cells. d Cyclin D1 stain of a sarcomatous malignant mesothelioma showing nuclear staining in $>75 \%$ of tumor cells

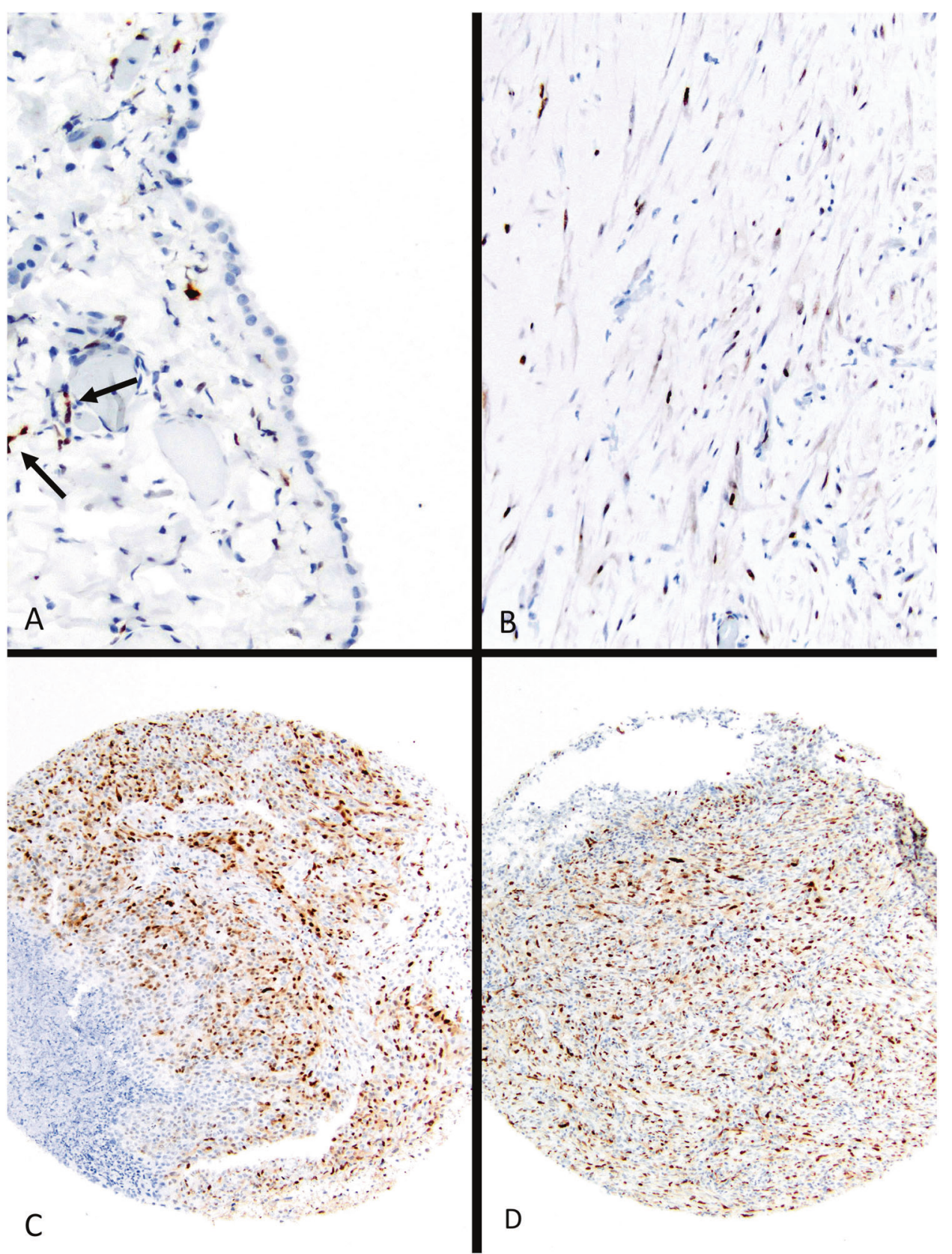

Table 2 BAP1 vs. Cyclin D1 in epithelial mesotheliomas

\begin{tabular}{lcl}
\hline & BAP1 lost & BAP1 retained \\
\hline Cyclin D1 $<50 \%$ staining & 3 & 10 \\
Cyclin D1 $>50 \%$ staining & 14 & 10 \\
\hline
\end{tabular}

(Table 2). Overall BAP1 was lost in 17/37 (46\%), 14 of which also showed $>50 \%$ cyclin D1 staining. BAP1 was lost in $3 / 13$ cases, in which cyclin D1 staining was $<50 \%$. MTAP staining was evaluable in 38 epithelial malignant mesotheliomas cases (Table 3). MTAP was lost in 14/38
Table 3 MTAP vs. Cyclin D1 in epithelial mesotheliomas

\begin{tabular}{lcl}
\hline & MTAP lost & MTAP retained \\
\hline Cyclin D1 $<50 \%$ staining & 4 & 10 \\
Cyclin D1 $>50 \%$ staining & 10 & 14 \\
\hline
\end{tabular}

(37\%), including ten, in which cyclin D1 staining was $>50 \%$, and four cases, in which cyclin D1 staining was $<50 \%$. Thus addition of MTAP to cyclin D1 staining increased sensitivity from 63 to $74 \%$, and additional of BAP1 to cyclin D1 increased sensitivity from 65 to $73 \%$. 


\section{Discussion}

The Hippo pathway is involved in control (largely restraint) of cell proliferation, apoptosis, and stress responses, and is in important in organ size development through maintenance of contact inhibition [11]. Although there is a large body of work on mechanisms and signalling networks of the Hippo pathway in malignancies, little has been published on the use of Hippo pathway components for diagnostic purposes. Some of the genes in the Hippo pathway are known tumor suppressor genes or oncogenes [11], and detection of abnormalities in this pathway in theory might be exploited for separating benign from malignant mesothelial processes.

Two important Hippo components that are frequently mutated or deleted in mesotheliomas are NF2 and LATS1/2 [12]. The published data on the diagnostic use of these Hippo pathway components in mesothelioma are limited and somewhat contradictory. Singhi et al. [10] showed that hemizygous loss of NF2 by FISH was associated with a worse prognosis in peritoneal mesothelioma; homozygous loss was not identified. Berg et al. [3] were unable to find any loss of NF2 by FISH in a series of pleural mesotheliomas, but more recently Kinoshita et al. [7] reported hemizygous loss in 53\% of a series of pleural mesotheliomas and used this test to separate reactive from malignant mesothelioma proliferations; again homozygous loss was not seen. Sheffield et al. [13] were unable to demonstrate differences in immunohistochemical staining of mesotheliomas and benign mesothelial reactions for Merlin (the protein product of NF2), LATS1/2, or YAP/TAZ, the latter the actual Hippo transcriptional coactivators.

The downstream effect of inactivating mutations or deletions in the Hippo pathway is increased YAP/TAZ signaling leading to increased transcription of a variety of genes, one of which is cyclin D1 [6,7]. When overexpressed, cyclin D1 itself drives rapid cell growth with bypass of normal inhibitory pathways [14].

Cyclin D1 immunohistochemical staining is a potentially attractive candidate for separating benign from malignant mesothelial proliferations because many laboratories already use cyclin D1 staining in working up lymphomas. Here we showed here that there are statistically significant differences in the expression of cyclin D1 in malignant mesotheliomas compared to reactive mesothelial proliferations. This difference is most obvious and appears to be most reliable in the separation of epithelial malignant mesotheliomas from reactive epithelial mesothelial proliferations because most of our cases of reactive epithelial mesothelial proliferations showed no cyclin D1 staining. None showed more than $25 \%$ of cells staining, and in fact most of the cases in the $1-25 \%$ category had only a small percentage of positive cells with none $>14.8 \%$ and all but one below $10 \%$. A similar staining pattern was seen in both tissue microarrays, suggesting that these differences are robust. As a practical proposition, we propose that an epithelial mesothelial proliferation with $>50 \%$ cyclin D1 staining should be viewed as malignant. At this cut-off in our study, cyclin D1 had an excellent specificity $(100 \%)$ and a moderate sensitivity $(57 \%)$ in distinguishing benign epithelial mesothelial proliferations from epithelial malignant mesotheliomas. However, for epithelial malignant mesotheliomas versus reactive epithelial mesothelial proliferations cyclin D1 staining is only useful with a high positive fraction; negative cases and cases in the 1-50\% staining category are not informative.

It should be borne in mind, also, that the Hippo pathway is dysregulated in a variety of malignant neoplasms including some nonsmall cell lung cancers [15], so that cyclin D1 overexpression is potentially present in many different tumors and is not, in and of itself, specific to mesotheliomas; thus confirmation that the process in question is mesothelial is required before using cyclin D1 staining.

Although the difference in distribution of staining between sarcomatous/desmoplastic malignant mesotheliomas and reactive spindle cell mesothelial proliferations is statistically significant, 33/51 (67\%) reactive spindle cell mesothelial proliferations were positive for cyclin D1, with many positive cases in the $1-25 \%$ range, some positive cases in the $26-50 \%$, and one positive case in the 51--75\% staining groups. An additional problem is that, without doing double immunostains, the separation of cyclin D1 vascular staining from mesothelial cell staining can be difficult when dealing with cells sitting in slit-like spaces between bundles of dense collagen. This should not be an issue in a cellular sarcomatous mesothelioma with a very high staining fraction such as Fig. 3d, but would be a problem when the differential is between a desmoplastic mesothelioma and paucicellular organizing pleuritis. An additional issue that we cannot address with microarrays is whether desmoplastic mesotheliomas show heterogeneous staining, which might result in false negatives. Thus we believe that cyclin D1 staining should not be used to try to separate desmoplastic mesotheliomas from organizing pleuritis.

We also examined the question of whether a combination of BAP1 and cyclin D1 or MTAP and cyclin D1 staining improves the separation of reactive epithelial mesothelial proliferations from epithelial malignant mesotheliomas (sarcomatous/desmoplastic malignant mesotheliomas rarely lose BAP1). We found that adding BAP1 or MTAP to cyclin D1 only produced around a $10 \%$ increase in sensitivity. In part this occurred because most cases in which BAP1 or MTAP was lost also showed $>50 \%$ cyclin D1 staining and only a small proportion of cases showed loss of BAP1 or MTAP and had $<50 \%$ cyclin D1 staining. However, the loss rates for both BAP1 (46\%) and particularly MTAP (37\%) in this particular set of cases are lower 


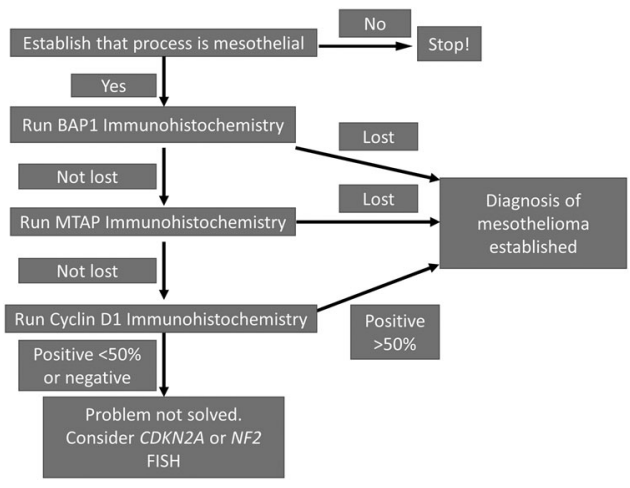

Fig. 4 One suggested approach to using BAP1, MTAP, and cyclin D1 immunostaining to separate epithelial mesotheliomas in the pleural cavity from reactive mesothelial proliferations when BAP1 and MTAP immunostaining are available. If BAP1 and/or MTAP are not available, starting with cyclin D1 is a reasonable alternative (see text)

than have been previously reported. The MTAP studies of Berg et al. [3], Chapel et al. [5], and Hida et al. [4] found sensitivities of 65,60 , and $46 \%$, so it is possible that evaluation of further cases might raise the overall sensitivity of combined cyclin D1/BAP1 or cyclin D1/MTAP stains. It is also worth noting that if these combinations are approached in the other direction, addition of cyclin D1 staining to BAP1 staining here increased sensitivity from 17/37 (46\%) to $27 / 37$ (73\%). Similarly, starting with MTAP staining, addition of cyclin D1 increased sensitivity from 14/38 (37\%) to $28 / 38$ (73\%).

Our results suggest a variety of approaches to utilizing cyclin D1 for separating mesotheliomas from reactive mesothelial proliferations, and this will depend on the morphology of the mesothelial process, the body cavity, and which antibodies are available in a given laboratory. Figure 4 shows a schematic approach if BAP1, MTAP, and cyclin D1 are all available. It's important to bear in mind that none of these markers individually has high sensitivity, but the combined sensitivity of BAP1 and MTAP for epithelial mesotheliomas in the pleural cavity is probably over $80 \%[3,4]$, and our current data suggest that adding cyclin D1 will raise this somewhat. Alternately, if only cyclin D1 is available, one could instead to start with that antibody and stop if $>50 \%$ staining is found. How these markers will work for epithelial mesotheliomas in the peritoneal cavity is less clear because there is no information for MTAP or cyclin D1 in peritoneal mesotheliomas. However, MTAP typically is codeleted with $C D K N 2 A$ [6], and the sensitivity of $C D K N 2 A$ FISH in the peritoneal cavity is low [2], so MTAP staining may not be productive.

For potential sarcomatous mesotheliomas (excluding desmoplastic mesotheliomas) one could apply the same scheme as shown in Fig. 4, but restricting cyclin D1 positivity to $>75 \%$ staining. Kinoshita et al. [16] reported a sensitivity of $>90 \%$ with a combination of BAP1 and
MTAP immunohistochemistry for sarcomatous mesotheliomas, although these authors also required that both markers exceed a specific cutoff for proportion of cells with loss of staining. However, our own experience and that of others is that BAP1 is generally very insensitive for sarcomatous mesotheliomas [2]. Alternatively, some combination of MTAP, cyclin D1, and PD-L1 staining might be useful for spindle cell mesothelial proliferations, with strong diffuse PD-L1 staining supporting a diagnosis of mesothelioma [17].

\section{Compliance with ethical standards}

Conflict of interest The authors declare that they have no conflict of interest.

Publisher's note Springer Nature remains neutral with regard to jurisdictional claims in published maps and institutional affiliations.

\section{References}

1. Chiosea S, Krasinskas A, Cagle PT, Mitchell KA, Zander DS, Dacic S. Diagnostic importance of 9p21 homozygous deletion in malignant mesotheliomas. Mod Pathol. 2008;21:742-7.

2. Churg A, Sheffield BS, Galateau-Salle F. New markers for separating benign from malignant mesothelial proliferations: are we there yet? Arch Pathol Lab Med. 2016;140:318-21.

3. Berg KB, Dacic S, Miller C, Cheung S, Churg A. Utility of methylthioadenosine phosphorylase compared With BAP1 immunohistochemistry, and CDKN2A and NF2 fluorescence In Situ hybridization in separating reactive mesothelial proliferations from epithelioid malignant mesotheliomas. Arch Pathol Lab Med. 2018;142:1549-53.

4. Hida T, Hamasaki M, Matsumoto S, Sato A, Tsujimura T, Kawahara K, et al. Immunohistochemical detection of MTAP and BAP1 protein loss for mesothelioma diagnosis: comparison with 9p21 FISH and BAP1 immunohistochemistry. Lung Cancer. 2017;104:98-105.

5. Chapel DB, Schulte JJ, Berg K, Churg A, Dacic S, Fitzpatrick C et al. MTAP immunohistochemistry is an accurate and reproducible surrogate for CDKN2A fluorescence in situ hybridization in diagnosis of malignant pleural mesothelioma. Mod Pathol. 2019. https://doi.org/10.1038/s41379-019-0310-0.

6. Chapel DB, Husain AN, Krausz T. Immunohistochemical evaluation of nuclear 5-hydroxymethylcytosine $(5-\mathrm{hmC})$ accurately distinguishes malignant pleural mesothelioma from benign mesothelial proliferations. Mod Pathol. 2019;32:376-86.

7. Kinoshita Y, Hamasaki M, Yoshimura M, Matsumoto S, Iwasaki A, Nabeshima K. Hemizygous loss of NF2 detected by fluorescence in situ hybridization is useful for the diagnosis of malignant pleural mesothelioma. Mod Pathol. 2019. In press.

8. Wu L, Dell'Anno I, Lapidot M, Sekido Y, Chan ML, Kohno M, et al. Progress of malignant mesothelioma research in basic science: a review of the 14th international conference of the international mesothelioma interest group (iMig2018). Lung Cancer. 2019;127:138-45.

9. Xiao GH, Gallagher R, Shetler J, Skele K, Altomare DA, Pestell $\mathrm{RG}$, et al. The NF2 tumor suppressor gene product, merlin, inhibits cell proliferation and cell cycle progression by repressing cyclin D1 expression. Mol Cell Biol. 2005;25:2384-94.

10. Singhi AD, Krasinskas AM, Choudry HA, Bartlett DL, Pingpank JF, Zeh HJ, et al. The prognostic significance of BAP1, NF2, and 
CDKN2A in malignant peritoneal mesothelioma. Mod Pathol. 2016;29:14-24.

11. Zygulska AL, Krzemieniecki K, Pierzchalski P. Hippo pathwaybrief overview of its relevance in cancer. J Physiol Pharmacol. 2017;68:311-35.

12. Hmeljak J, Sanchez-Vega F, Hoadley KA, Shih J, Stewart C, Heiman D, et al. TCGA research network. Integrative molecular characterization of malignant pleural mesothelioma. Cancer Discov. 2018;8:1548-65.

13. Sheffied BS, Lorette J, Shen Y, Marra MA, Churg A. Immunohistochemistry for NF2, LATS1/2, and YAP/TAZ fails to separate benign from malignant mesothelial proliferations. Arch Pathol Lab Med. 2016;140:391.
14. Qie S, Diehl JA. Cyclin D1, cancer progression, and opportunities in cancer treatment. J Mol Med. 2016;94:1313-26.

15. Han Y. Analysis of the role of the Hippo pathway in cancer. J Transl Med. 2019;17:116.

16. Kinoshita Y, Hamasaki M, Yoshimura M, Matsumoto S, Sato A, Tsujimura T, et al. A combination of MTAP and BAP1 immunohistochemistry is effective for distinguishing sarcomatoid mesothelioma from fibrous pleuritis. Lung Cancer. 2018;125:198-204.

17. Derakhshan F, Ionescu D, Cheung S, Churg A. Use of programmed death ligand-1 (PD-L1) staining to separate sarcomatoid malignant mesotheliomas from benign mesothelial reactions. Arch Pathol Lab Med. 2019. In press. 